\title{
ARTÍCULO RETIRADO: Diseño y construcción de un molde permanente utilizando un software por elementos finitos
}

\section{(RETRACTED: Design and construction of a permanent mold using finite element software)}

\author{
Fausto Oviedo Fierro ${ }^{1}$, Alexy Vinueza Lozada ${ }^{2}$
}

\section{Nota del Comité Editorial:}

El presente artículo fue retirado luego de comprobarse que había sido publicado también en otra revista, violando las normas de Enfoque UTE. EI Comité Editorial de Enfoque UTE toma esta decisión honrando las directivas de la comunidad científica, pese a tener una cesión de derechos firmada por el autor correspondiente.

\section{Editorial Board Notice:}

This article was retracted upon receiving confirmed evidence of double publication, therefore disrespecting the journal's code of conduct. This Editorial Board takes this decision to honor the scientific community guidelines, in spite of having a Transfer of Copyright Agreement signed by the corresponding author.

\section{Resumen:}

El proyecto fue desarrollado con especificaciones técnicas de un método de elementos finitos para el cálculo de las variables de campo dentro de un contorno del molde de estudio y eligiendo un modelo matemático apropiado con la incorporación de software. El desarrollo fue una aplicación físico-practica seleccionando una pieza de aluminio a ser construida, y se analiza las funciones y solicitaciones a łas que está sometida. Se diseña el molde metálico para la producción de las mencionadas piezas con la ayuda del software VULCAN basado en el Método de Elementos Finitos (MEF). La simulación se realiza en las tres etapas del proceso de fusión: llenado, solidificación y, enfriamiento. En el llenado se considera que existen velocidades que no produzcan mayores turbulencias y que las piezas se llenen completamente. En la Solidificación se analiza que el sistema de alimentación sea óptimo para que solidifique en última instancia y no lo hagan las piezas a producir, evitando así el defecto de falta de material en la cavidad del molde (rechupes). En el enfriamiento se analiza la presencia de deformaciones y tensiones residuales. Paralelamente se validan los resultados de manera analítica. Con esta información se define la geometría final del molde metálico y se confirma la aleación con la que se construirá. Posteriormente se utiliza el sistema CAD-CAM-CAE para el diseño y desarrollo del molde, y así llevar a cabo la construcción. En el molde terminado se vierte la aleación de aluminio seleccionada para obtener las piezas (mancuernas), y finalmente realizar las pruebas y conclusiones.

Palabras clave: MEF, molde metálico, sistema de alimentación, rechupes, moldura, mancuerna, solidificación.

Abstract:

\footnotetext{
${ }^{1}$ Escuela Politécnica Nacional, Quito - Ecuador (fausto.oviedo@epn.edu.ec)

${ }^{2}$ Universidad Tecnológica Equinoccial, Facultad de Ciencias de la Ingeniería, Quito - Ecuador (vlaf92847@ute.edu.ec)
} 
The project was developed with specifications of a finite element method for the calculation of the field variables within a mold contour by choosing an appropriate mathematical model which permitted to incorporate simulation software. The development was a physical-practical application for a piece of aluminum that will be built, and consists in the analysis of the functions and stresses to which it is submitted. The metal mold is designed specifically for the mentioned pieces using "VULCAN" which is based on the Finite Element Method (FEM). The simulation is performed in three stages of the fusion process: filling, solidification and cooling. The filling is considered slow enough so that there are not expected to be greater turbulences and that the pieces are completely filled. Solidification is analyzed as an optimal process, avoiding defects of lack of material in the mold cavity (shrinkage). In the cooling stage, deformations and residual stresses are analyzed. At the same time, every result is validated analytically. With this information the final geometry of the metal mold is defined and the alloy which will be used is confirmed. Subsequently, a CAD-CAM-CAE system is used for the design and development of the mold, and the aluminum pieces (knobs) obtained are tested.

Keywords: FEM, die, power system, shrinkage, mold, knob, solidification.

\section{Referencia tecnológica}

EI MEF como modelo matemático estructurado se desarrolla en la primera mitad del siglo pasado, Galerkin proporciona una base muy sólida para el MEF pero no fue sino hasta la década de 1940, con Courant que se considera el inicio del método de los elementos finitos. Con esta base ingenieros aeronáuticos desarrollaron métodos matriciales para el análisis de las estructuras de fuselajes para soportar grandes cargas asociadas a altas velocidades. Esto lo hicieron sin el beneficio de los ordenadores modernos. El verdadero impulso del MEF se da con el avance de la informática. En nuestro medio existen varios software en estudios de MEF para la simulación en piezas fundidas (NovaFlow \& Solid, ViewCast, CastCAE, MAVIS, Vulcan-GID, ANSYS, etc.) (The Stockton Casting Company, 1994; Engineering Software Solutions, 2004; ANSYS, 2013; Solidification \& Flow Analysis, 2012) y êl uso de estos programas está poco aplicado en el campo de la fundición de piezas.

Este trabajo presenta las claras ventajas del uso de simuladores de colado y solidificación de metales para hacen posible el uso con ahorros en el proceso de manufactura a nivel latinoamericano.

2. Método de elementos finitos

Un aspecto fundamental para una correcta simulación es una clara comprensión del procedimiento implementado por el software a utilizar. El objetivo de este método es el cálculo de las variables de campo dentro de su contorno. Para ello se procede a elegir un modelo matemático apropiado que como característica principal debe tener continuidad, y luego se procede a dividir el dominio en un número determinado (finito) de elementos, esta división se la determina por las características intrínsecas del fenómeno que estemos analizando y de su forma. Con esta base se desarrolla matrices para cada elemento y luego se las ensambla en una matriz de rigidez global, con las características principales de esta matriz cuadrática y simétrica. 
Definido el concepto matemático del fenómeno se desarrollan los siguientes pasos (Hutton, 2004)

Preprocesado. En el que se define la geometría del problema, el tipo de malla y de elementos discretos que se selecciona, las propiedades físicas de los elementos, las condiciones de borde.

Solución. Definido el fenómeno de estudio se usa un software, el cual ensambla las matrices y calcula las variables desconocidas.

Posprocesado. El software no presenta los valores en magnitudes, y corresponde darle elorden correspondiente para que los resultados sean presentados en sus magnitudes. Es importante chequear el equilibrio del sistema definiendo con criterio ingenieril, en el que conviene résolver con una carga crítica en la mínima sección de la pieza y así determinar los factores de seguridad para que las soluciones sean las correctas (Shigley, 2005).

\section{Software basados en el MEF para la fundición de metales}

De los más conocidos en un primer grupo son: CapCast EKK, Flow-3DCast, MagmaSoft, Mavis, NovaCast, ProCast, QuickCast, Simtec, SolidCast, TherCasty, Vulcan-GID. Dentro del segundo grupo se debe destacar a ANSYS y ABAQUS. que solucionan los principales problemas que se presentan en el fenómeno de llenado, solidificación y enfriamiento de una moldura producida en coquilla y en molde perdido.

Para el diseño del molde metálico se utiliza el Software VULCAN, este es un producto de la empresa Quantech, iniciada en el año 1987 en la Universidad Politécnica de Catalunya cuando se crea el Centro Internacional de Métódos Numéricos en Ingeniería (CIMNE).

\section{Diseño de las piezas a producir}

\subsection{Selección de las piezas y su material}

Dado el proceso de manufactura por fundición y la demanda de piezas con su forma se seleccionan mancuernas para cierre de ventanas de una edificación. Estas son las piezas a ser producidas para el diseño y construcción del molde en el presente trabajo mostrado en la Figura 1.
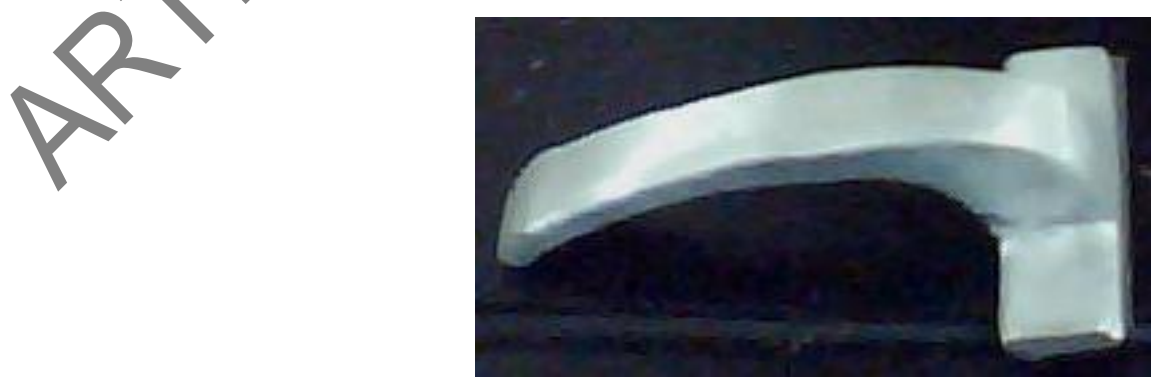

Figura 1. Ejemplo de mancuerna para cerrar ventanas en una edificación.

Para determinar el tipo de material de las mancuernas se realiza el siguiente análisis: función, restricciones y variables libres (Ashby, 2004). 
Función. La mancuerna sirve para garantizar el cierre de ventanas de casas. Puede estar en ambientes corrosivos; debe ser de baja densidad, de fácil instalación y tener una aceptable resistencia mecánica.

Restricciones. La mancuerna debe ser de una aleación metálica que pueda ser producida por fundición y debe tener bajo costo.

Variables libres. La longitud y el espesor pueden variar de acuerdo al diseño de tal manera que cumpla con los requerimientos.

En base a estos criterios y a la experiencia nacional en la producción de este tipo de pieźas se elige como material una aleación de aluminio con buena colabilidad, designada por la Aluminium Association de USA como la aleación 324.0, la cual es recomendada y cuya composición es: Si: 7\%; Cu: 0.5\%;Mg: 0.7\% (Hufnagel, 1995).

\subsection{Selección de la forma final de la pieza}

Diseñar es formular un plan para llenar una necesidad que habrá de ser satisfecha. Esto abarca varias disciplinas de Ingeniería Mecánica, tales como análisis de esfuerzos, ciencia de materiales, ciencias térmicas y de fluidos, con lo cual se va a identificar los problemas de desempeño de la pieza.

Con estos criterios se presenta a continuación en la Tabla 1 las consideraciones para el diseño de la pieza a fabricar.

Tabla 1. Consideraciones para el diseño de piezas fabricadas por colado de metal fundido (Titov, Stepanov, 1981).

Diseño para piezas de colado de metal fundido

1. Se deben tomar consideraciones sobre el grosor de paredes. Las partes más delgadas se solidifican antes por lo que deben buscarse espesores uniformes.

2. Evitar acabados esquinados para eliminar la concentración de esfuerzos durante el moldeo y la solidificación.

3. Se deben considerar los claros de la pieza respecto al molde, necesarios para una mejor expulsión.

4. Evitar formas que impidan la expulsión de la pieza o hagan necesario el uso de mecanismos complejos para sacar las piezas.

5. Se requiere delinear la línea de partición del molde en la figura, con asistencia de un software o pericia del diseñador.

6. Buscar la simetría de la pieza. Geometrías más complejas significan mayores costes del molde y tiempo de fabricación.

Se presenta 3 alternativas de diseño de la mancuerna, las cuales evolucionan desde la actual pieza presente en el mercado hasta una pieza que cumple con las consideraciones de la Tabla 1 Se elije la alternativa 3 que cumple con todas las consideraciones, y se representa en la Figura 2 


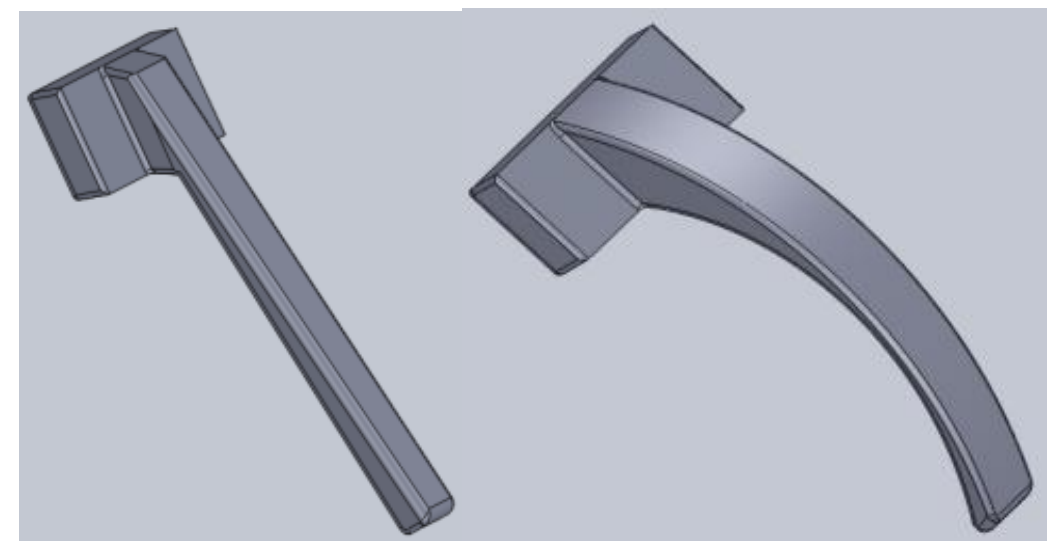

Alternativa 1 y 2 de la mancuerna
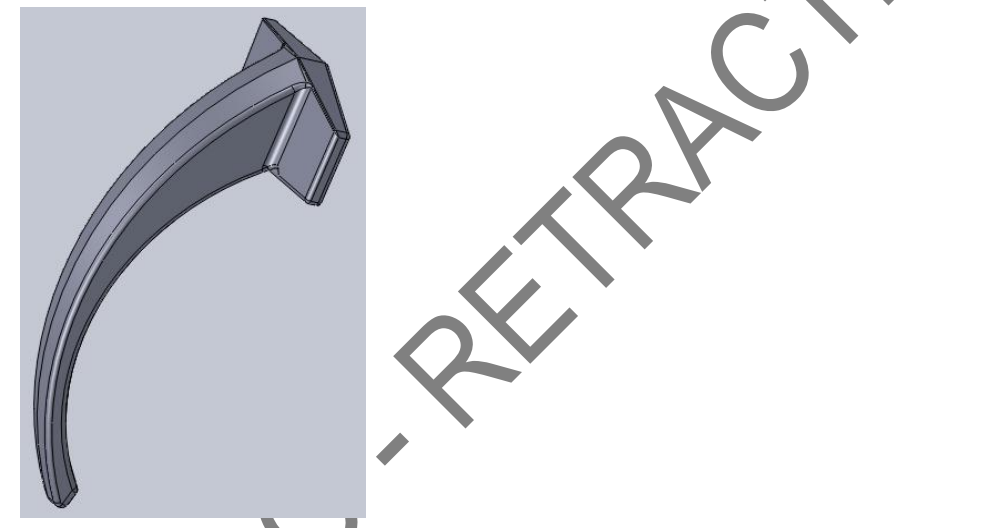

Figura 2. Esquema de la Mancuerna C.

\subsection{Análisis de esfuerzos por el MEF}

Alternativa 3

Para la práctica de simulación se tóma una viga en voladizo como representación del estudio estático para un diagrama esfuerzo cortante y momento flector de la Figura 3 y así utilizar el modulo estructural estático del software ANSYS, el cual desarrolla y presta soporte a la ingeniería para predecir cómo funcionará y reaccionará determinado producto bajo un entorno real.
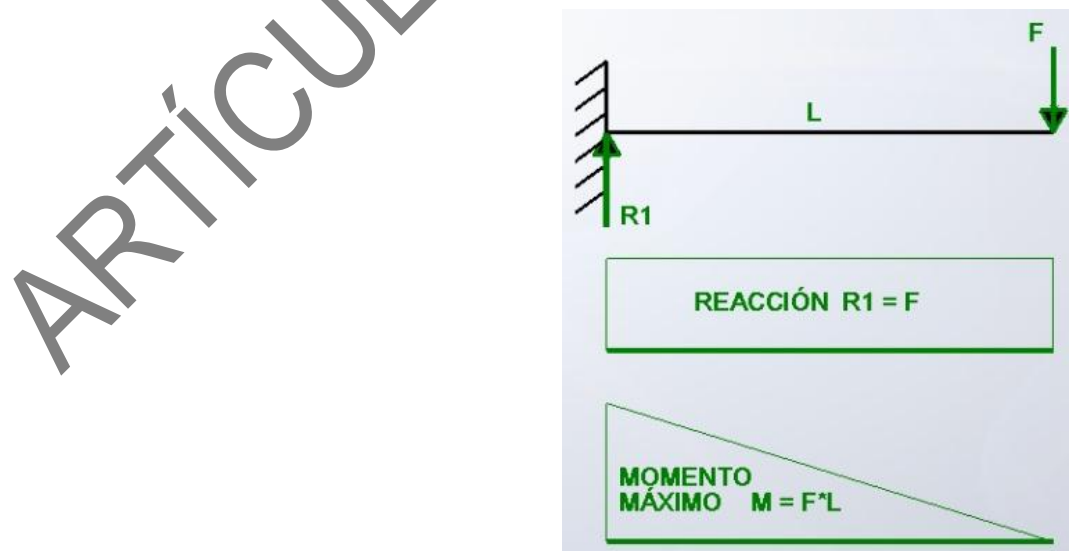

Figura 3. Diagrama de esfuerzo cortante y momento flector.

Se parte de un análisis estático de una viga en voladizo. La tensión máxima $[\sigma]$ se representa por: 


$$
\sigma \leq \frac{F L}{W_{\min }}
$$

[Ecuación 1]

Donde:

F es la carga máxima sobre el eje voladizo

$L$ es la longitud del eje voladizo

$W_{\min }$ es el momento resistente de la sección de menor área

El esfuerzo aplicado sobre la sección transversal viene dado por:

$$
\sigma=\frac{F}{A_{t}}
$$

Dónde:

$F$ : Fuerza ejercida $[N]$

$A_{t}:$ Área de la sección transversal $\left[\mathrm{mm}^{2}\right]$

El factor de seguridad [n] se define por la siguiente ecuación:

$$
n=\frac{s}{\sigma}
$$

[Ecuación 3]

Dónde:

S: Resistencia a la fluencia del aluminio = 34.4 [Mpa] (Shigley, 2005 \& Hufnagel, 1995)

$\sigma$ : Esfuerzo aplicado [Mpa]

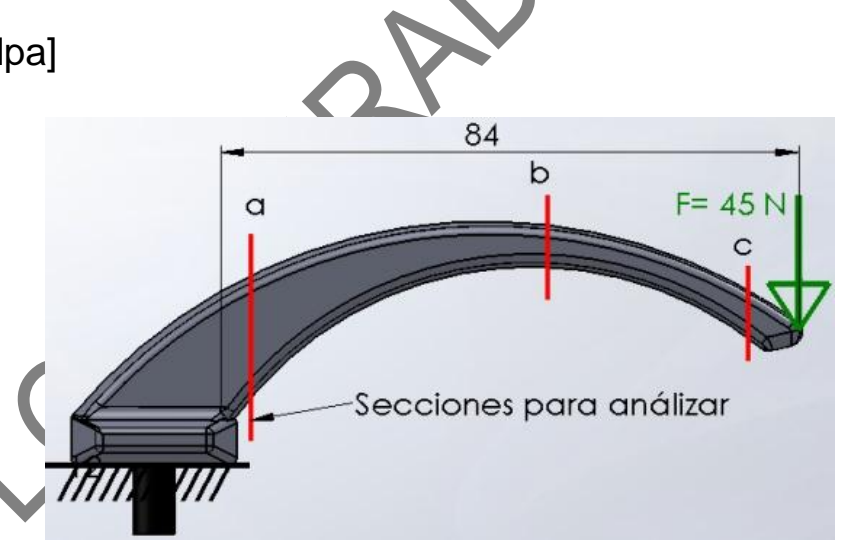

Figura 4. Diagrama de Equilibrio.

De la Figura 4 se obtienen los siguientes resultados de equilibrio de fuerzas y momentos.

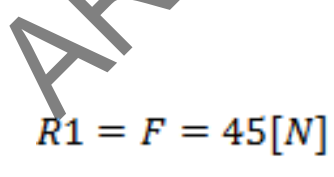

$$
\sum F_{x}=0
$$$$
R 1-F=0
$$

$$
M=F * L
$$$$
M_{M A X}=45 * 84=3780[N * m m]
$$

Utilizando la Ecuación 2 y la menor área de sección transversal en [b] de la Figura 4 que corresponde a $49\left[\mathrm{~mm}^{2}\right]$ se obtiene el esfuerzo aplicado sobre esa sección.

$$
\sigma=\frac{45[\mathrm{~N}]}{49\left[\mathrm{~mm}^{2}\right]}=0.92[\mathrm{Mpa}]
$$


Con la Ecuación 3 se determina el factor de seguridad en el punto donde la sección transversal es la más pequeña.

$$
n=\frac{34.4[\mathrm{Mpa}]}{0.92[\mathrm{Mpa}]} \quad n=37
$$

Se concluye que la pieza con las medidas definidas soporta las condiciones de trabajo tanto en la simulación de ANSYS como con el cálculo analítico. Definiéndose las dimensiones finales mostradas en la figura 5 .

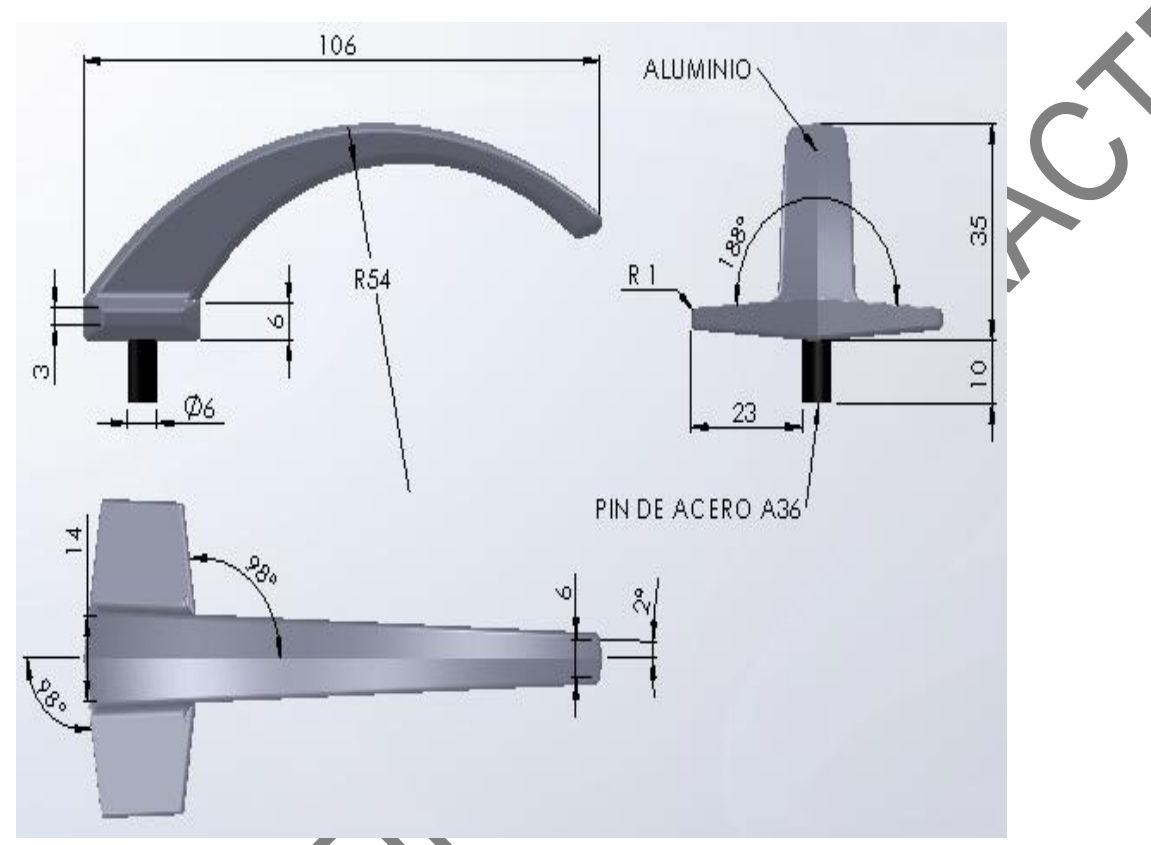

Figura 5. Dimensiones de la mancuerna.

\section{Diseño del molde metálico}

Las ventajas de su uso, son la gran precisión y calidad del acabado superficial de las piezas obtenidas. Además, son muy económicos cuando se producen grandes cantidades de piezas. Existen varios tipos de mơldes metálicos utilizados para la fabricación de piezas principalmente no ferrosas.

El tipo de molde metálico diseñado y construido es a gravedad. Esta determinación se la hace debido a que este tipo de molde es el más adaptable para ser usado en los talleres y fábricas de fundición del país y es el adecuado para el tipo de pieza a producir (Beeley, 2001). Se procede a elegir un molde metálico para producir mancuernas de aluminio para cerrar ventanas, la colada de aluminio oscila entre 680 a $750^{\circ} \mathrm{C}$ de temperatura.

A continuación en la Figura 6 se presenta el flujo del proceso de diseño del molde metálico: 


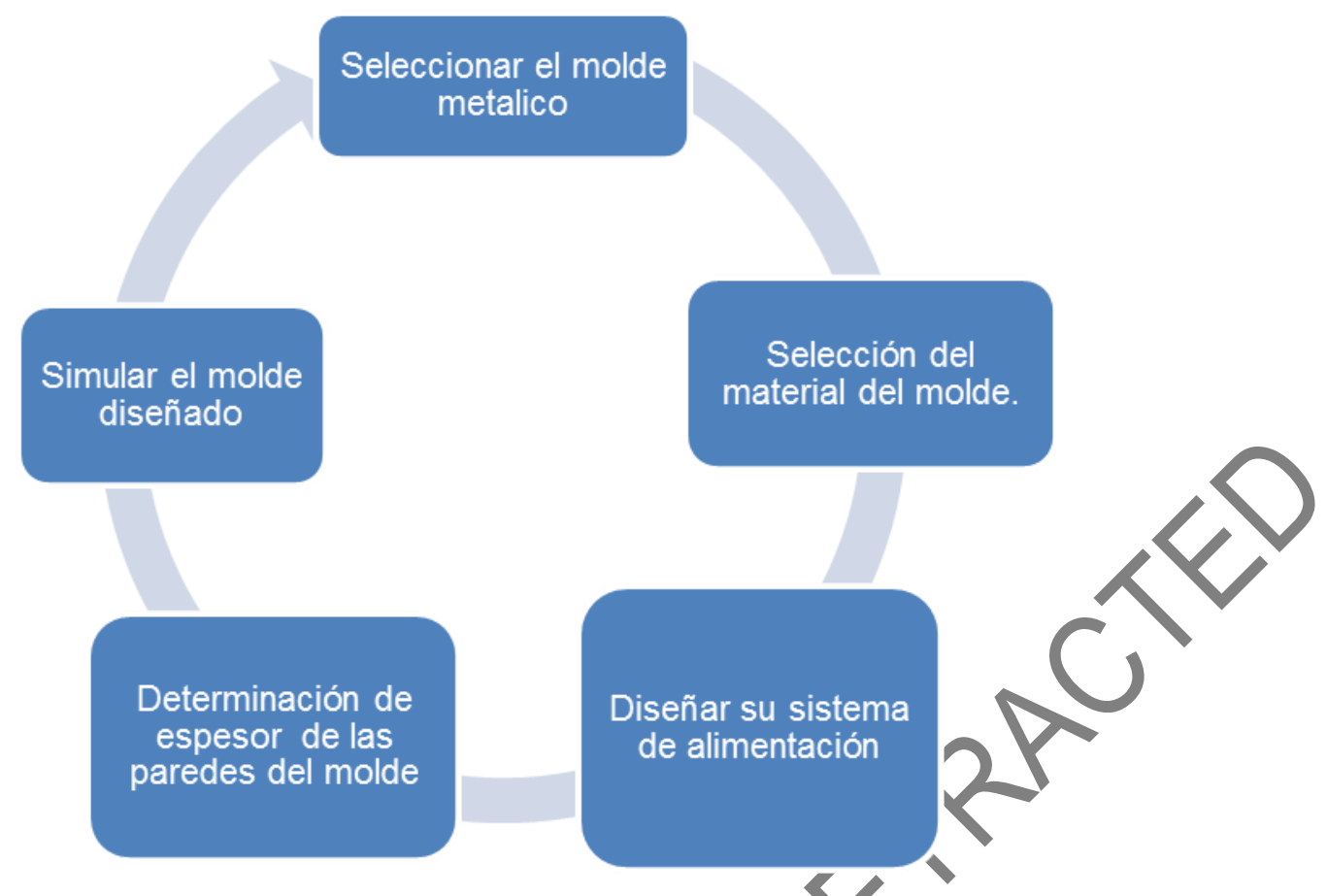

Figura 6. Proceso de diseño del moldemetálico.

\subsection{Selección del molde metálico.}

La necesidad de producción es de cuatro mancuernas a la vez con una masa total de $200 \mathrm{gr}$. y una producción de 50 piezas por hora. La vida útił de la coquilla es para una fabricación de 10000 mancuernas (ASM Metal Hand book, 1988)

El ingreso del metal es al mismo nivel para las cuatro mancuernas como se señala en las alternativas de diseño en la Figura 7

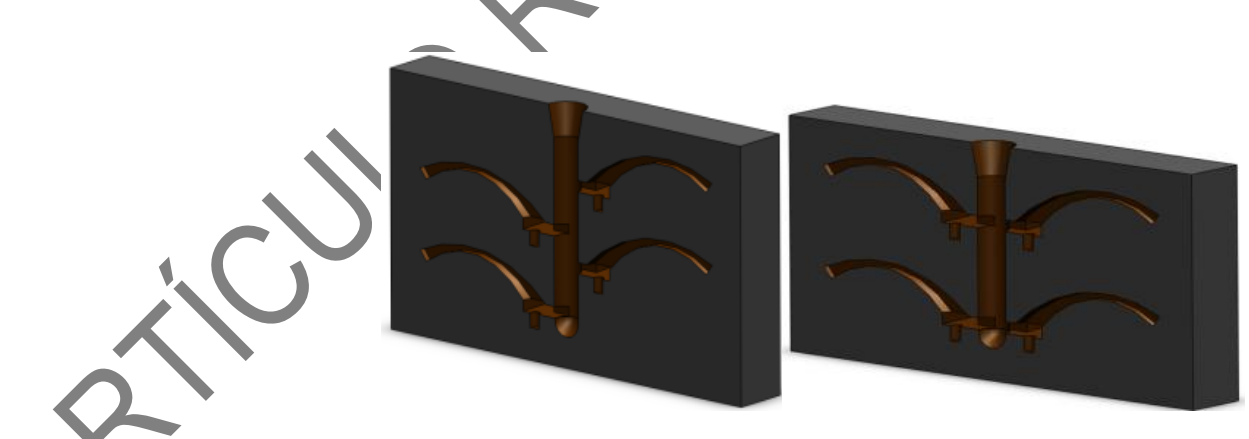

Alternativas 1 y 2 (no elegidas) 


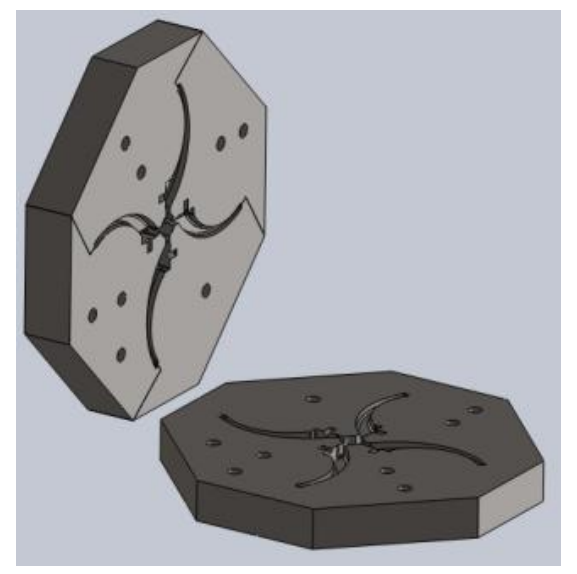

Figura 7. Alternativa 3(elegida).

Para seleccionar el material del molde se considera que debe ser un acero para trabajos en caliente que posea resistencia a grietas por calentamiento, tenacidad en caliente, resistencia al impacto y que admita refrigeración con agua (Ashby, 2004).

El Acero ideal para este uso sería el AISI: P20 (mejorado), según DIN: 40CrMnNiMo8-6-4, en aceros BÔLHER: M238. Sin embargo por aspectos de costo se toma la recomendación del (ASME Metals Handbook, 1988) y se elige el acero A36 comercial.

\subsection{Determinación del espesor de las paredes}

El espesor de las paredes del molde mostrado en la Figura 8, depende del tamaño de las piezas que se vayan a colar. Un molde de paredes demasiado gruesas con relación a la pieza tiene una inercia térmica demasiado grande, lo que retrasa su calentamiento y posteriormente su enfriamiento, mientras que un molde delgado se calienta demasiado en cada llenado pero se enfría con rapidez en cada vaciado.

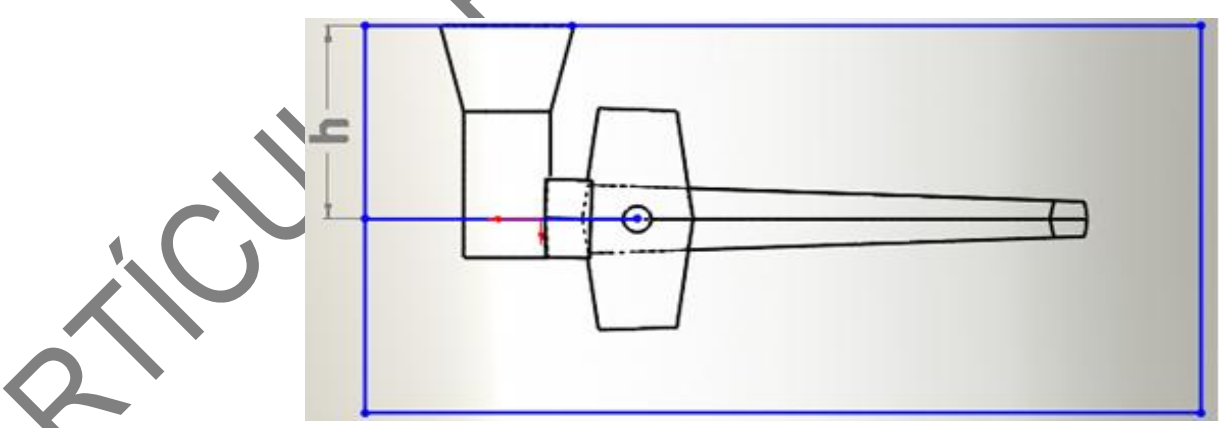

Figura 8. Espesor de pared.

El criterio que se considera es la altura del montante de alimentación. Para determinar esta altura se utiliza la fórmula de Navas (1990)

$$
V m \geq \frac{V p \times R E}{U-R E}
$$

[Ecuación 4]

Donde:

$V m=$ Volumen de la Mazarota

$V p=$ Volumen de la mancuerna 
$U=\%$ de utilización del metal

$R E=$ Rechupe específico del metal en la mazarota (\%)

$R E=7.2$

$U=14$

Determinándose que

$h=45 \mathrm{~mm}$

Esta es la altura del montante de alimentación y se toma como el valor para el espesor de la coquilla.

\subsection{Diseño del sistema de alimentación}

El sistema de alimentación debe garantizar un buen llenado de las piezas, una buenà alimentación mientras solidifica, evitar la retención de gases en el interior de las piezas, uná suficiente presión metalostática y así posibilitar una fácil separación de los canales de alimentación (Navas,1990).

Se define el área del canal vertical o montante y el canal de alimentación con el método de círculos inscritos (Taylor, 1959) mostrado en la Figura 9, que fuego se analiza con el Software VULCAN de elementos finitos.

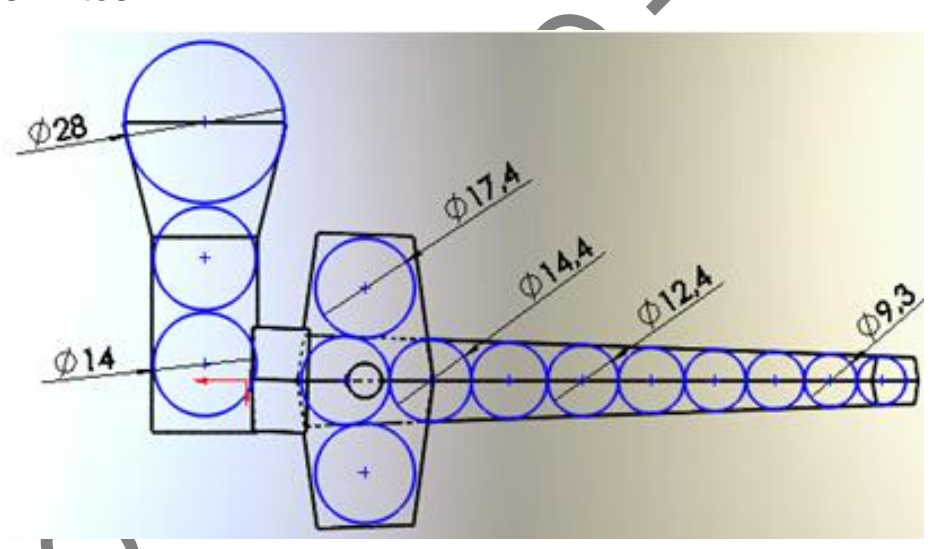

Figura 9. Determinación de $A_{M}$ por el método de círculos inscritos

\subsection{Simulación del llenado}

EI Programa VULCAN permite realizar la simulación y su análisis en tres etapas: llenado, solidificación térmica y enfriamiento termo-mecánico. Se procede a simular siguiendo el orden indicado:

1. Se importa la geometría.

2. Se crea las capas molde y moldura. Es importante elegir una capa para el molde y otro para el sistema de alimentación y pieza, en este caso se ha llamado Molde y Moldura respectivamente y se representan en la Figura 10. 


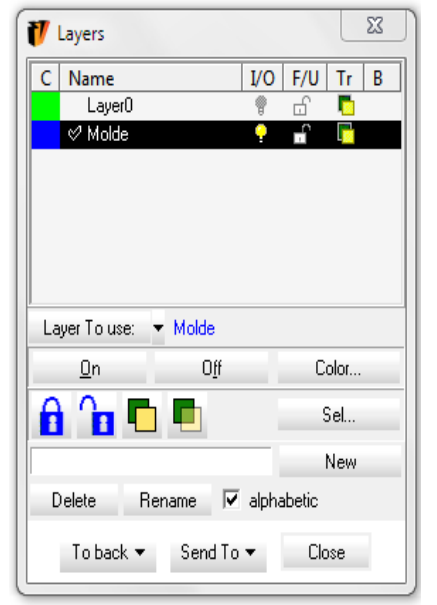

Figura 10. Separación en diferentes capas al molde y a la moldura

3. Se crean superficies en los planos inferior y superior del Molde:

4. Se crea el orificio de ingreso de metal en la superficie superior del molde mostrado en la Figura 11.

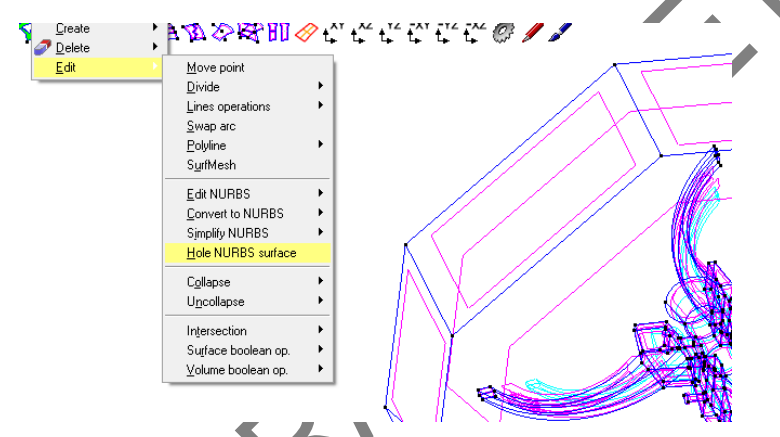

Figura 11. Creación del ôrificio én la superficie superior del molde

5. Se crea el volumen del molde.

6. Se selecciona el tamaño delos elementos y se malla.

7. Se define el tipo de problema en el software que se detalla en la Figura 12.

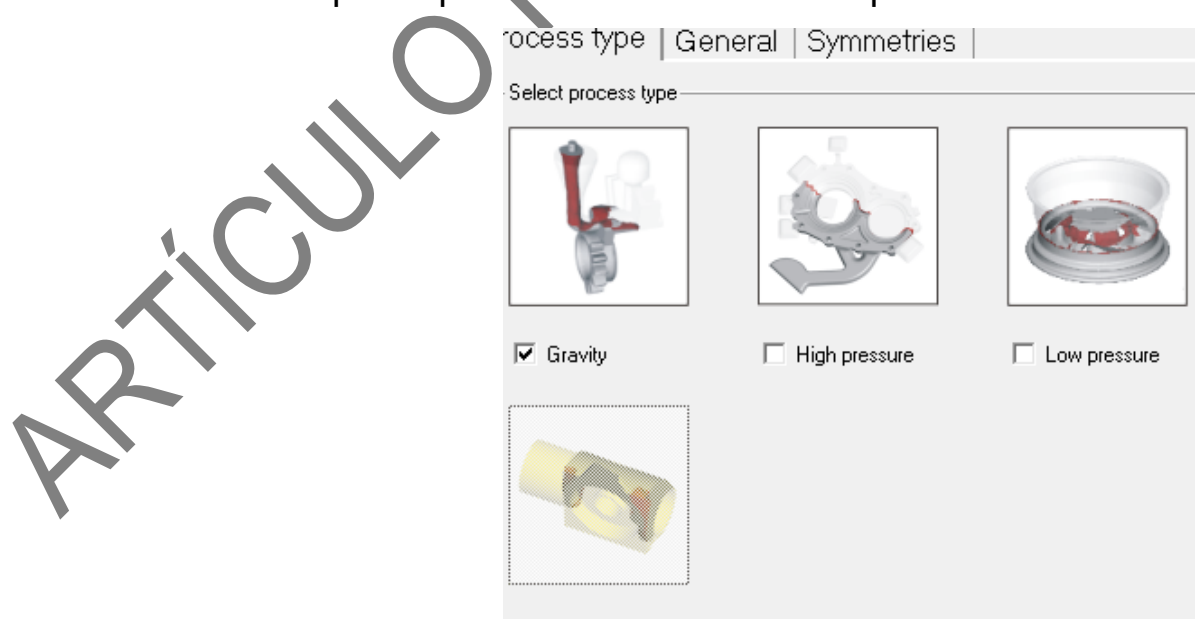

Figura 12. Selección del tipo de problema

8. Se define los componentes del proceso de fundición: es necesario seleccionar los materiales del molde como está indicado en la Figura 13. 


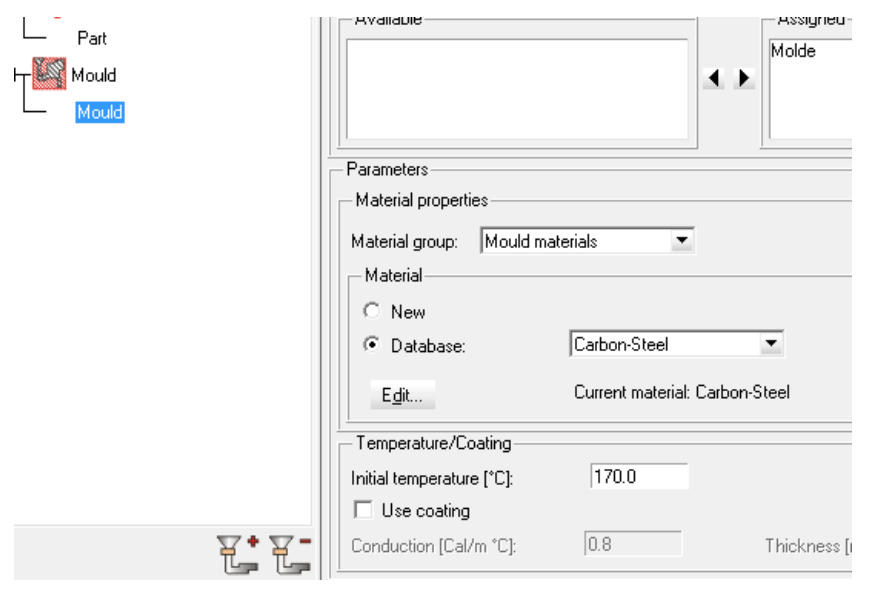

Figura 13. Selección del material para el molde y moldura

9. Se define el tipo de cálculo a realizar. En este caso el Llenado.

10.Se procede aguardar el Proyecto y se ordena calcular.

11. Se desarrolla el cálculo.

12. Se pasa al pos proceso y se carga los resultados obtenidos según la Figura 14.

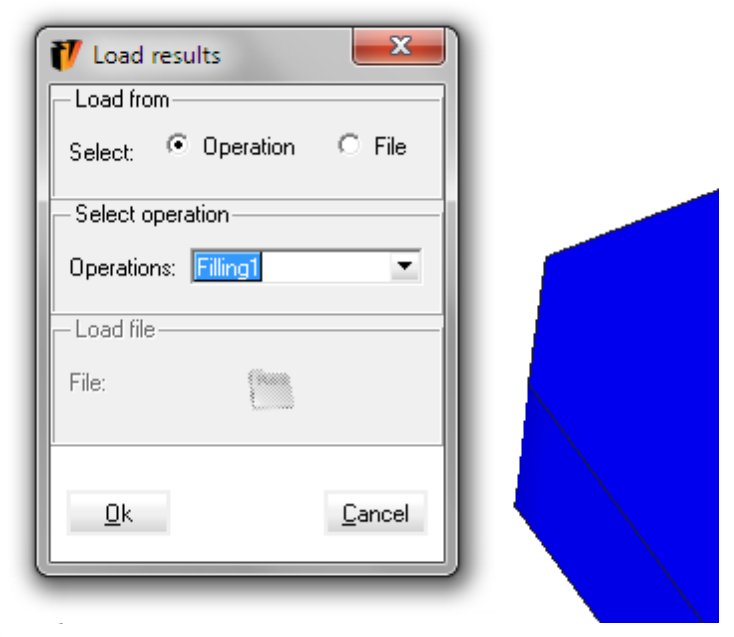

Figura 14. Carga de resultados obtenidos

Finalmente se procede a la lectura e interpretación de los resultados y en este caso los más importantes son las zonas donde se tienen los últimos aires mostrados en la Figura 15.

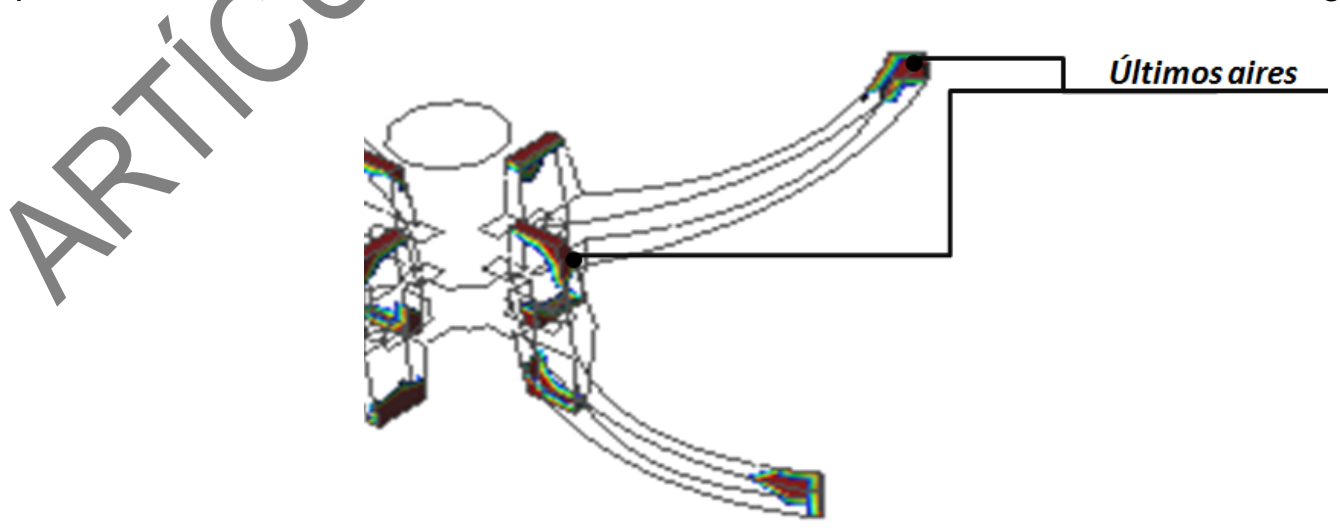

Figura 15. Zonas de presencia de los últimos aires

\subsection{Simulación de la solidificación térmica}


Realizando el mismo procedimiento hasta el paso 8 seguido en la simulación de llenado se procede con la Solidificación Térmica. Se prueba inicialmente con un diámetro del montante de $7 \mathrm{~mm}$, luego con 9mm, y así observar los últimos sólidos en la moldura de la Figura 16.

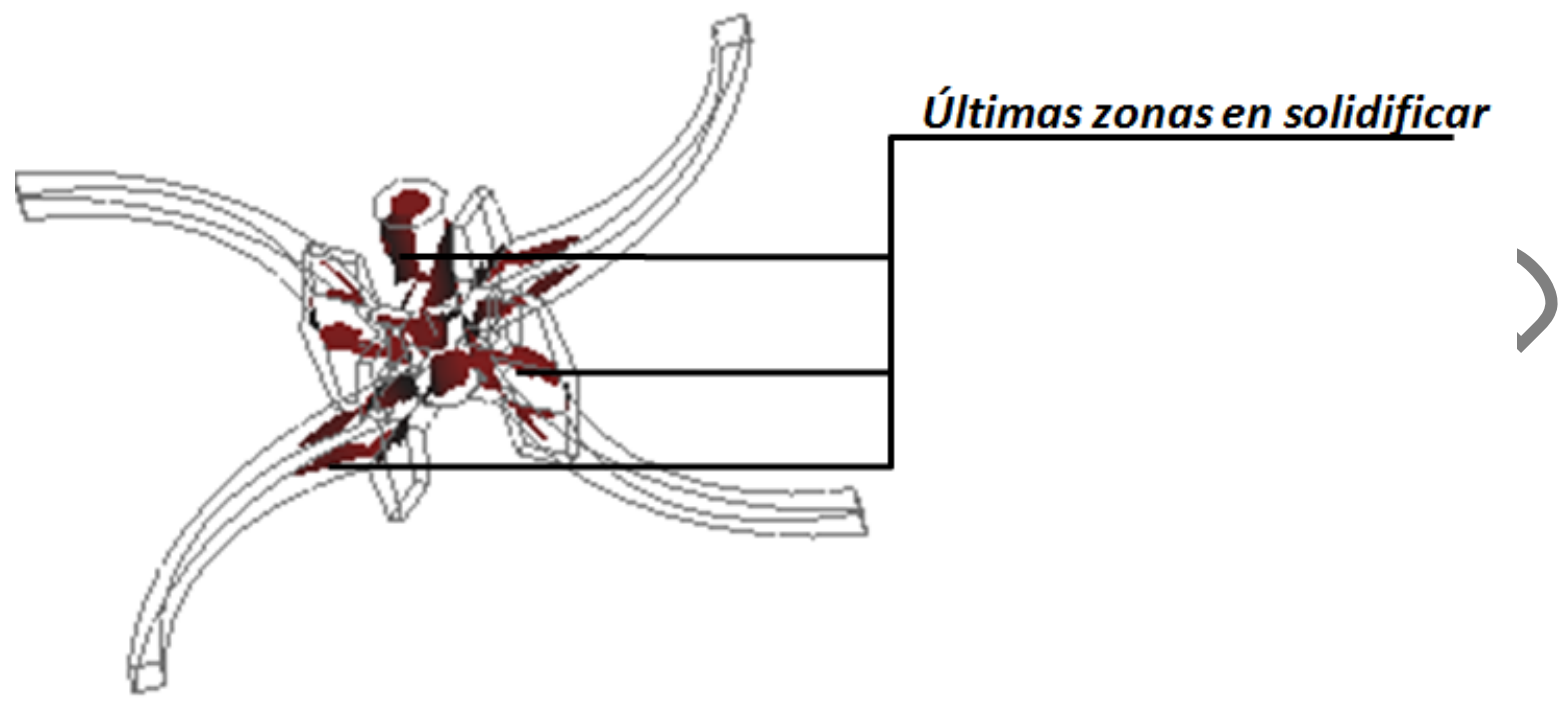

Figura 16. Últimos sólidos en la moldura con radio de $7 \mathrm{~mm}$

Con este nuevo valor se procede a simular nuevamente. Adicionalmente, se perfecciona el mallado haciéndolo más fino en la zona de contacto del sistema de alimentación con la pieza obteniéndose que los últimos sólidos se trasladan al montante, aunque quedan aún algunos en la pieza, como se muestra en la Figura 17.

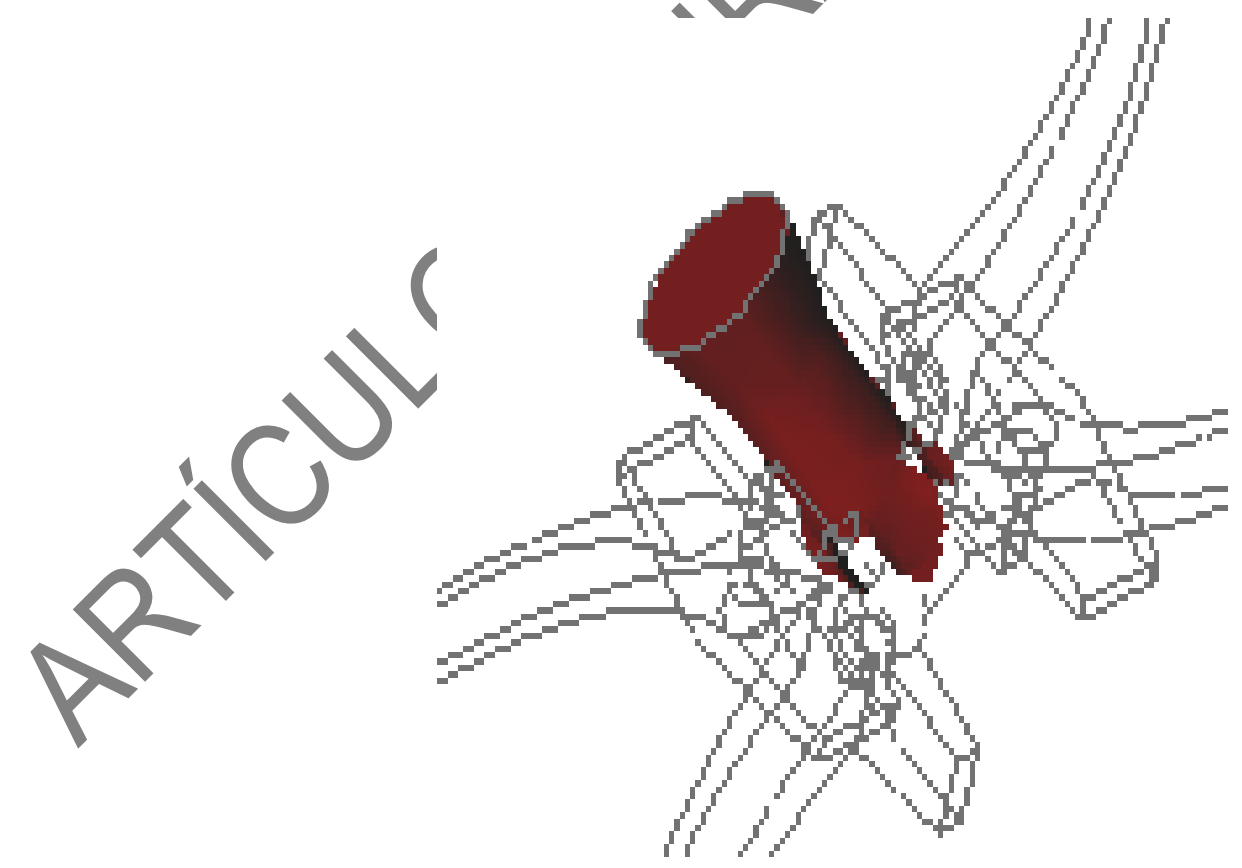

Figura 17. Últimos sólidos en la moldura con radio de $10 \mathrm{~mm}$

Se simula con 10mm y se observa que la última fracción de solido es en el montante. 
Otro factor importante a analizar es el defecto Niyama y es la relación basada en el gradiente de temperatura y la velocidad de enfriamiento desarrollada por el investigador japonés Dr. Niyama, en el estudio de la predicción de rechupe del acero, el cual es un criterio aplicado al software de simulación de fundición que anticipa la presencia de rechupes en una determinada zona.

Se presenta a continuación en la Tabla 2 , un cuadro de resumen de los resultados obtenidos en la simulación:

Tabla 2. Resumen de las simulaciones

\begin{tabular}{|c|c|l|l|c|}
\hline $\begin{array}{c}\text { RADIO DEL } \\
\text { MONTANTE }\end{array}$ & Llenado & $\begin{array}{l}\text { Últimos sólidos } \\
\text { existentes }\end{array}$ & Defecto Niyama & $\begin{array}{l}\text { Tensiones } \\
\text { Residuales }\end{array}$ \\
\hline $7 \mathrm{~mm}$ & Bueno & $\begin{array}{l}\text { En montantey } \\
\text { Pieza }\end{array}$ & $\begin{array}{l}\text { Alto en montante y } \\
\text { medio en pieza }\end{array}$ & Nulo \\
\hline $9 \mathrm{~mm}$ & Bueno & $\begin{array}{l}\text { Más en montante } \\
\text { que en pieza }\end{array}$ & $\begin{array}{l}\text { Alto en montante y } \\
\text { medio en pieza }\end{array}$ & Nulo \\
\hline $10 \mathrm{~mm}$ & Bueno & Soloen montante & $\begin{array}{l}\text { Alto en montante y } \\
\text { Bajoen pieza }\end{array}$ & Nulo \\
& & & &
\end{tabular}

\subsection{Validación de resultados}

Con la simulación se han seleccionado las áreas del montante y de los canales de alimentación. Para validar estas áreas se realiza el cálculo utilizando el método de Y. Dubinski [NAVAS, 1990]

$$
\sum A a=G /(T v * K) \mathrm{cm}^{2}
$$

[Ecuación 5]

Donde:

$\mathrm{A} a=$ Area del Montante

$\mathrm{G}=$ masa del liquido que pasa por el sistema de alimentación en $\mathrm{Kg}$.

$\mathrm{K}=$ Velocidad específica de vertido $\mathrm{Kg} /\left(\mathrm{cm}^{2} . \mathrm{s}\right)$

Tv = Tiempo de vertido
$T,=S \sqrt{G} \mathrm{~S}$.
[Ecuación 6]

Aplicando estas ecuaciones se obtiene el siguiente radio del montante:

$r=0.96 \mathrm{~cm}=9.6 \mathrm{~mm}$, aproximando:

$r=10 \mathrm{~mm}$

Para el canal de alimentación se considera colado a presión con una relación de áreas:

Area $=A a^{\star} 7 / 16=137.4 \mathrm{~mm}^{2}$

Siendo la altura del canal de $20 \mathrm{~mm}$ el espesor del canal "e" será:

$\mathrm{e}=137,4 \mathrm{~mm}^{2} / 20 \mathrm{~mm}=6,87 \mathrm{~mm}$, aproximando este valor se tiene

$\mathrm{e}=7 \mathrm{~mm}$ 
Los dos valores $r=10 \mathrm{~mm}$ y e $=7 \mathrm{~mm}$ confirman los valores alcanzados con la simulación con el software VULCAN.

\section{Modelación y construcción de la coquilla}

Para el mecanizado del molde se utiliza el software CAM para Maquinas Herramientas de Control numérico computarizado (CNC) y que se representa en la Figura 18.

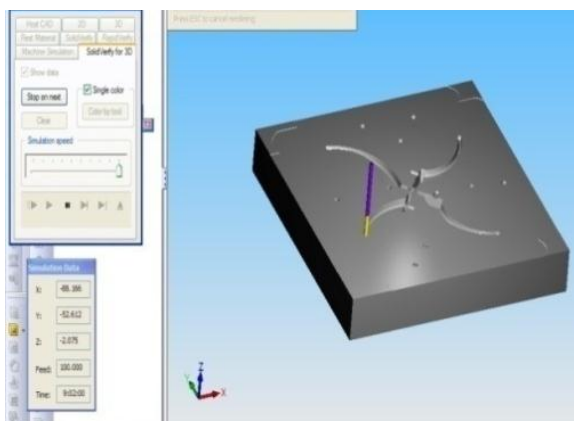

Figura 18. Trayectoria de la herramienta en la simulación del software CAM

La metodología de fabricación de este tipo de moldes es aplicable en máquinas CNC, por lo que se efectúan todas las operaciones para que sean realizadas por dichas máquinas y su resultado se visualiza en la Figura 19.

\section{Pruebas}

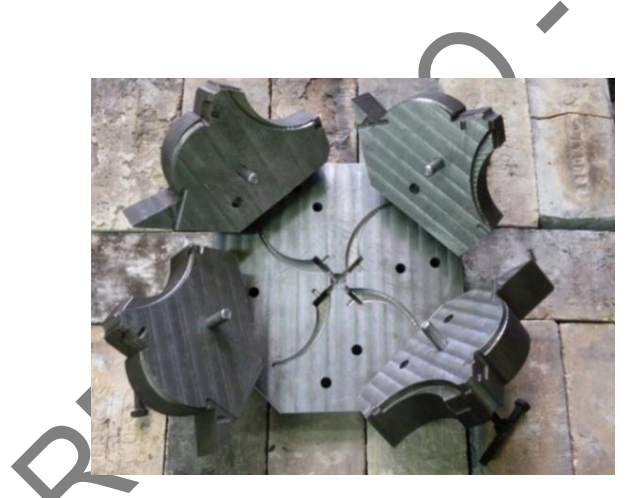

Figura 19. Detalle de Ensamblaje Molde Permanente

Se selecciona la aleación de aluminio AA 333.0 con $10 \%$ de silicio y $3 \%$ de cobre elegida en el diseño, se eleva a la temperatura en un horno a gas GLP en crisol de hierro fundido revestido, se llega a la temperatura de fusión en 1 hora, seguidamente se recalienta el material hasta $740^{\circ} \mathrm{C}$, se desgasifica, se procede a colar a una temperatura de $720^{\circ} \mathrm{C}$, para asi obtener la siguiente Tabla 3 . En la Figura 20, se visualiza la moldura obtenida luego de la colada y su posterior enfriamiento.

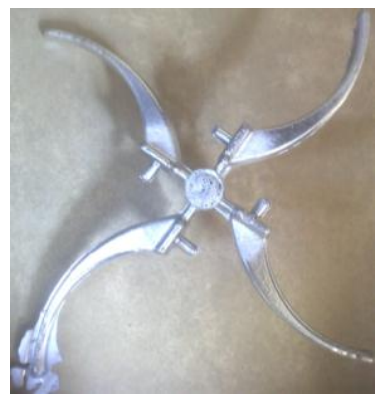

Figura 20. Moldura obtenida 
Tabla 3. Resultados Finales

\begin{tabular}{|c|c|c|c|c|c|c|c|}
\hline $\begin{array}{l}\frac{\pi}{0} \\
\frac{\pi}{0} \\
0\end{array}$ & 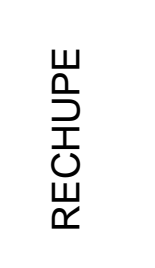 & 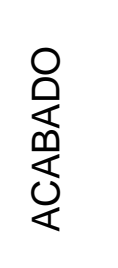 & $\begin{array}{l}\text { Temp. } \\
\text { Colada }\end{array}$ & $\begin{array}{c}\text { Temp. } \\
\text { Superficial }\end{array}$ & $\begin{array}{l}\text { Temp. } \\
\text { Media }\end{array}$ & 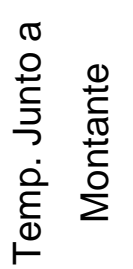 & $\begin{array}{l}\text { O } \\
\frac{0}{0} \\
0 \\
\frac{0}{0} \\
0 \\
0 \\
\end{array}$ \\
\hline & & & ${ }^{\circ} \mathrm{C}$ & ${ }^{\circ} \mathrm{C}$ & ${ }^{0} \mathrm{C}$ & ${ }^{0} \mathrm{C}$ & $s$ \\
\hline 1 & Pequeño & Bueno & 723 & 41 & 76 & 50 & 90 \\
\hline 2 & Ninguno & Medio & 724 & 52 & 72 & 60 & 90 \\
\hline 3 & Ninguno & Bueno & 730 & 54 & 74 & 97 & \\
\hline 4 & Ninguno & Bueno & 725 & 56 & 77 & 111 & \\
\hline 5 & Ninguno & Bueno & 722 & 58 & 75 & & 90 \\
\hline
\end{tabular}

\subsection{Análisis del proceso utilizado y de los resultados obtenidos}

- El software VULCAN es una gran ayuda para el diseño de moldes permanentes. Los resultados que se obtienen reducen el tiempo de diseño y permite eliminar errores en el sistema de alimentación.

- Con el uso del Software se evita perdidas de material y de trabajo, permite directamente construir un molde útil una vez simulado.

- Con la simulación en el software se tiene una clara orientación para los errores que se presentan en el período de pruebas luego de construida la coquilla.

- Se utilizó un software CAD paralos dibujos, lo cual simplifica todo el proceso, porque estos mismos gráficos sirven de planos para la elaboración de los códigos $\mathrm{G}$ para su posterior construcción en el Centro de Maquinado CNC.

- El momento de las pruebas, se tomo muy en cuenta que los valores de: temperatura de colado, altura de vertido y temperatura a la que llega la coquilla, sean similares a los considerados en la simulación.

\section{Conctusiones}

Este trabajo es pionero en el uso de software de simulación para la producción de moldes permanentes en el país, dando así un aporte importante a la tecnificación de la producción de piezas fundidas.

El uso de este método en la industria fundidora nacional llevará a mejorar el nivel tecnológico en el diseño de moldes permanentes.

La simulación es una herramienta muy útil pero no remplaza al cálculo analítico ni a las consideraciones técnicas que el diseñador debe tomar. 
Es muy importante tener las mismas consideraciones de borde tanto en el momento de simular como cuando se realiza el cálculo analítico y en las pruebas.

Se realizaron dos pruebas obteniéndose en la segunda ocasión las piezas completas de buena calidad.

\section{Recomendaciones}

Dada la precisión de las herramientas actuales (En este caso el hilo por corte de electroerosión) es importante revisar si los planos de unión dan la suficiente salida de aire.

Se debe continuar profundizando en el uso del Software VULCAN y realizar capacitación del uso de otros Software para la simulación en fundición de igual o mayor capacidad de resolución.

Es necesario profundizar en el estudio y comprensión del fenómeno físico, especialmente la dinámica del metal líquido y la transferencia de calor, tanto en estado estable como inestable.

Se debe desarrollar investigación y cálculos en software matemáticos que permitan la solución de los modelos que representan el fenómeno.

\section{Bibliografía}

The Stockton Casting Company Ltd (1994), Computer aided mould filling and casting solidification simulation. Recuperado el 15 de julio de 2012, de http://www.stocktoncastings.co.uk/simulation.htm

The casting Technology International (2004), E-Technology Used to Optimise Product Design and Reduce Inventory Levels. Recuperado el 20 de Julio del 2012, de http://www.castingstechnology.com/news.asp?nsKeywords=Allen+case+study\&xcCommand =search

Enginnering Software Solutions (2009). Recuperadoel 25 de Julio del 2012, de www.quantech.es/vulcan.aspX

ANSYS (2013). Recuperado el 22 de Enero del 2013, de www.ansys.com

Solidification \& Flow Analysis (2012). Recuperado el 12 de Abril del 2012, de http://www.metal-technologies.com/SolidificationAnalysis.aspx

Hutton D. (2004). Fundamental of finite element analysis (pág.486). USA: Mac. Graw Hill

ASME Metals Hand Book. Vol 15 (1988). Sociedad Americana de Ingenieros Mecánico. USA.

Ashby M (2004). Materials Selection in Mechanical Design (pág.302). USA: EH.

Hufnagel W. (1995). Manual del aluminio (pág.456). España: Reverté S.A

Shigley M. (2005). Diseño de Ingeniería Mecánica(pág. 876). USA: Mc Graw-Hill.

Titov N. y Stepanov Y. (1981). Tecnología del proceso de fundición (pág.460). Moscú: Mir.

Flemings (1974). Solidification Processing (pág.355). USA: McGraw-Hill. 
Beeley P.(2001, 731p). Foundry Technology (pág.731). Oxford: Butterworth-Heinemann José Ramón, Alique López (1981). Control Numérico CNC. Barcelona: Boixareu. Navas y Otros (1990). Métodos de Cálculo en Fundición (pág.184). CUBA: ISTH. Taylor H. (1959). Foundry Engineering (pág. 407). USA.

Institute Of Physics (2003). Solidification and casting (pág.420). Oxford: UK. B Cantor. Campbell J. (2003). Castings. The new metallurgy of cast metals (pág.328). Oxford: Butterworth Heinemann

Garrido C \& Calentano D. (2002). Simulación tridimensional del proceso de solidificación de una aleación de aluminio en un molde compuesto. CONAMET/SAM-SIMPOSIO 6. 